\title{
Vibration analysis of an inhomogeneous string for damage detection by wavelet transform
}

\author{
Chung-Jen Lu*, Yu-Tsun Hsu \\ Department of Mechanical Engineering, National Taiwan University, No. 1 Roosevelt Rd. Sec. 4, \\ Taipei 10617, Taiwan
}

Received 28 February 2001; received in revised form 7 January 2002

\begin{abstract}
In this paper we present a new method based on the wavelet transform for the detection of structural damage. It is assumed that the vibration signal of the original structure without any defect is already known. In the case when defects are present, the vibration signals of the defected structure are then recorded. After comparing the discrete wavelet transforms of these two sets of vibration signals in the space domain, we are not only able to detect the presence of defects, but also their number and location as well. To test the effectiveness of the proposed method, we use a uniform string as the original undamaged structure. To simulate the defects of the structure, we attach several point masses and springs on the string. Numerical result shows that even a minor localized defect can induce significant changes in the wavelet coefficients of the vibration signals. Furthermore, the maximum change of the wavelet coefficients occurs in the proximity of the defect. These results indicate that the proposed method could potentially be an alternative tool in the structural damage detection. (C) 2002 Elsevier Science Ltd. All rights reserved.
\end{abstract}

\section{Introduction}

The real-time condition monitoring for structural and machine damage detection is an important research topic [1-5]. Most of the methods currently in use for this purpose are based on the processing of structural vibration signals. A commonly used method for vibration signal processing is modal analysis [4,6-12]. The main idea behind damage detection schemes that use modal data is that a change in the system due to damage will manifest itself as changes of the natural frequencies and the associated mode shapes. However, these methods may exhibit low sensitivity to defects in the early stage of development and/or poor diagnostic capability [4,6,7].

\footnotetext{
${ }^{*}$ Corresponding author. Tel.: +886-2-2362-7686.

E-mail address: cjlu@ccms.ntu.edu.tw (C.-J. Lu).
} 
A defect in the early stage, e.g. a crack, is localized in space and the induced vibration signals are not stationary. This may be the main reason why the modal data, which are global and stationary, are not sensitive to the early localized damage. On the other hand, the wavelet analysis, which possesses multi-resolution, provides a promising method to solve this problem. Several researchers employed the wavelet transform for the diagnosis of rotary machinery [13-16]. They applied the wavelet transform in the time-domain to analyze the vibration signals measured at convenient locations. Due to the constant rotation speed, the time-domain data can be easily related to the angular position of the shaft, which makes it possible to pinpoint the location of the defect. However, it is generally difficult to locate the defect from the time-domain results without knowing the dynamic characteristics of the system.

In this paper we present a method based on the wavelet transform in the space domain for identifying the existence and location of structural damage. In order to concentrate on the studying of the effectiveness of this method, a uniform string is used to represent the original undamaged system, while a spring and some mass points are attached to the uniform string to simulate different kinds of defects. The vibration signals of the damaged system are processed with wavelet transform and the results are compared with those of the original system for damage detection.

\section{Wavelet interpolation}

It can be shown that under some conditions, a function $u(x) \in L^{2}(R)$ can be expressed as the superposition of the wavelets $\psi_{k}^{j}(x)[17]$ :

$$
u(x)=\sum_{j=-\infty}^{\infty} \sum_{k=-\infty}^{\infty} c_{k}^{j} \psi_{k}^{j}(x),
$$

where $\psi_{k}^{j}$ are generated by dilating and translating the mother wavelet $\psi(x)$,

$$
\psi_{k}^{j}(x)=a_{j}^{-1 / 2} \psi\left(\left(x-b_{k}^{j}\right) / a_{j}\right),
$$

in which $a_{j}$ and $b_{k}^{j}$ are the dilation and translation parameters, respectively. For clarity of discussion, wavelets corresponding to the same $j$ are called the wavelets of the $j$ th level of resolution.

In this paper we use the discrete wavelet transform in the space domain to analyze the vibration of a string with finite length. Let $u(x)$ denote the displacement of the string at a certain instant. If $u(x)$ is defined on a closed interval $\Omega \equiv\left[x_{l}, x_{r}\right]$, the dilation and translation parameters in Eq. (2) are taken as

$$
a_{j}=2^{-j} a_{0}, \quad b_{k}^{j}=\left(x_{r}+x_{l}\right) / 2+k a_{j} b_{0},
$$

where $a_{0}=2^{-L}\left(x_{r}-x_{l}\right) / b_{0}, L \in Z$. For a proper choice of $L$ all the levels below $j=0$ can be approximated by a constant, so for the numerical approximation all these lower levels can be excluded [18]. Note that for any $j$ there are values of $k$ such that the wavelets are centered outside of the domain $\Omega$. These wavelets are called external wavelets; the other wavelets whose centers are located within the domain are called internal wavelets. For appropriately defined wavelets, which are either with compact support or decay fast enough away from their center, there are always a finite number of external wavelets whose influence inside of the domain must be accounted for. Thus at any level 
$j$ there is a subset of integers

$$
\left\{Z_{\Omega}^{j}:-2^{L+j-1}-N_{l}, \ldots, 2^{L+j-1}+N_{r}\right\}
$$

such that for $k \in Z_{\Omega}^{j}$ the wavelet $\psi_{k}^{j}$ affects significantly the interior of the domain $\Omega$. In Eq. (4), $N_{l}$ and $N_{r}$ are the numbers of external wavelets on each side of the domain. Therefore, Eq. (1) can be further simplified as

$$
u^{J}(x)=\sum_{j=0}^{J} \sum_{k \in Z_{\Omega}^{j}} c_{k}^{j} \psi_{k}^{j}(x),
$$

where the levels $j=0$ and $j=J$ correspond, respectively, to the coarsest and the finest scales.

The collocation method developed by Vasilyev et al. [18,19] is adopted in this paper for determining the wavelet coefficients $c_{k}^{j}$. Since there are in total $2^{L+j}+N_{l}+N_{r}+1$ wavelet coefficients at the $j$ th level of resolution, we need the same number of collocation points to determine these wavelet coefficients. The strategy developed in Ref. [18] is employed to construct the collocation points.

\section{Physical model-non-uniform string}

In order to concentrate on the study of the effectiveness of the proposed method, a relatively simple model, a string is employed here. A uniform string is used to represent the original undamaged system. Since a defect in the early stage may induce changes of the local density and/or flexibility, a spring and some mass points are superimposed to the uniform string to simulate different kind of defects. The wavelet transform is adopted to analyze the vibration signals of the string for studying the relation between the wavelet coefficients and the superimposed elements.

The equation of motion of a string with density $\rho(x)$, length $l$, under a constant tension $T$ is

$$
T \frac{\partial^{2} u}{\partial x^{2}}-K \delta(x-\bar{x}) u=\rho(x) \frac{\partial^{2} u}{\partial t^{2}}
$$

where $K$ and $\bar{x}$ indicate the stiffness and location of the attached spring, respectively. The density of the string can be expressed as

$$
\rho(x)=\rho_{0}+\sum_{i} m_{i} \delta\left(x-x_{i}\right)
$$

where $\rho_{0}$ is the density of the uniform string, $m_{i}$ is the amount of the mass superimposed at $x=x_{i}$. In this paper, we let $T=1, \rho_{0}=1$ and $l=2$. The total mass of the uniform string is $\rho_{0} l=2$.

For a uniform string with both ends fixed, the $n$th natural frequency is $\omega_{n}=n \pi / 2$ and the associated mode $\phi_{n}=\sin (n \pi x / 2)$. These modes are used to express the displacement $u(x, t)$ of the non-uniform string as

$$
u(x, t)=\sum_{i=1}^{\infty} c_{i}(t) \phi_{i}(x) .
$$

Then Galerkin's method is used to determine the coefficients $c_{i}(t)$ and the displacement at the collocation points. Finally, the wavelet coefficients are determined by the method developed in Ref. [18]. 


\section{Results and discussion}

We first consider the case of a point mass $m$ attached to the center of a uniform string. In this case, the density of the string is given by $\rho(x)=\rho_{0}+m \delta(x-1)$. We use the point mass to represent a highly localized damaged part of the original system. Fig. 1 shows the displacement of the string at time $t=0.2$ of various values of $m$ under the same initial conditions: $u(x, 0)=\sin (\pi x / 2)$ and $\dot{u}(x, 0)=0$. As can be seen from the figure, the deflection of the uniform string $(m=0)$ at $t=0.2$ and that of the non-uniform string with $m=0.02$ are almost identical. In other words, when the added point mass is $1 \%$ of the mass of the uniform string, it is difficult to identify the existence of the point mass by merely monitoring the deflection. Then we use the method described in Section 2 to determine the wavelet coefficients of these two systems at different levels of resolution. In order to demonstrate the effectiveness of the proposed method, we further reduce the point mass to $m=0.005(0.25 \%$ of the mass of the uniform string). The well-known "Mexican hat" wavelet function, $\psi(x)=\left(x^{2}-1\right) \exp \left(-x^{2} / 2\right)$, is employed as the mother wavelet to illustrate the method. This choice is arbitrary. Other wavelets that have compact support or decay fast enough can also be used. We set the highest level of resolution to $J=5$. Fig. 2 shows the wavelet coefficients at collocation points from the 2nd to the 5th level, where solid and dashed lines indicate results of the uniform $(m=0)$ and non-uniform $(m=0.005)$ strings, respectively. Although the deflections of these two systems are almost identical, their wavelet coefficients are quite different from each other. Moreover, it can be observed that at each level the maximum difference occurs at the center of the string, where the point mass is located. For the convenience of discussion, we denote the region where the difference between the two sets of wavelet coefficients is significant as the "deviation region". As can be seen from the figure, the "deviation region" at each level is centered at the location of the point mass and its width decreases as the level of resolution increases. Therefore, the "deviation region" can be used as a good indication regarding the existence and location of the point mass.

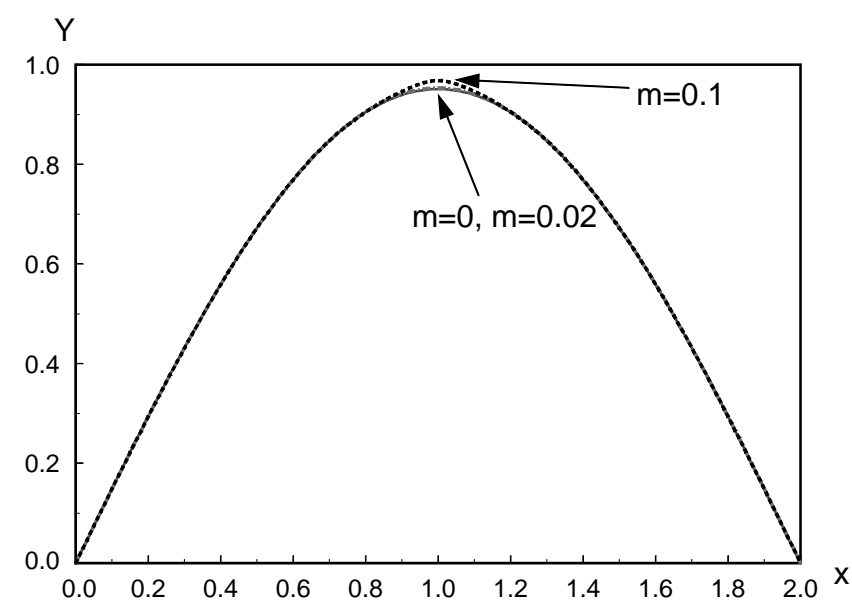

Fig. 1. Deflections at $t=0.2$ of a uniform string with a central concentrated mass of various magnitudes under the same initial conditions. 

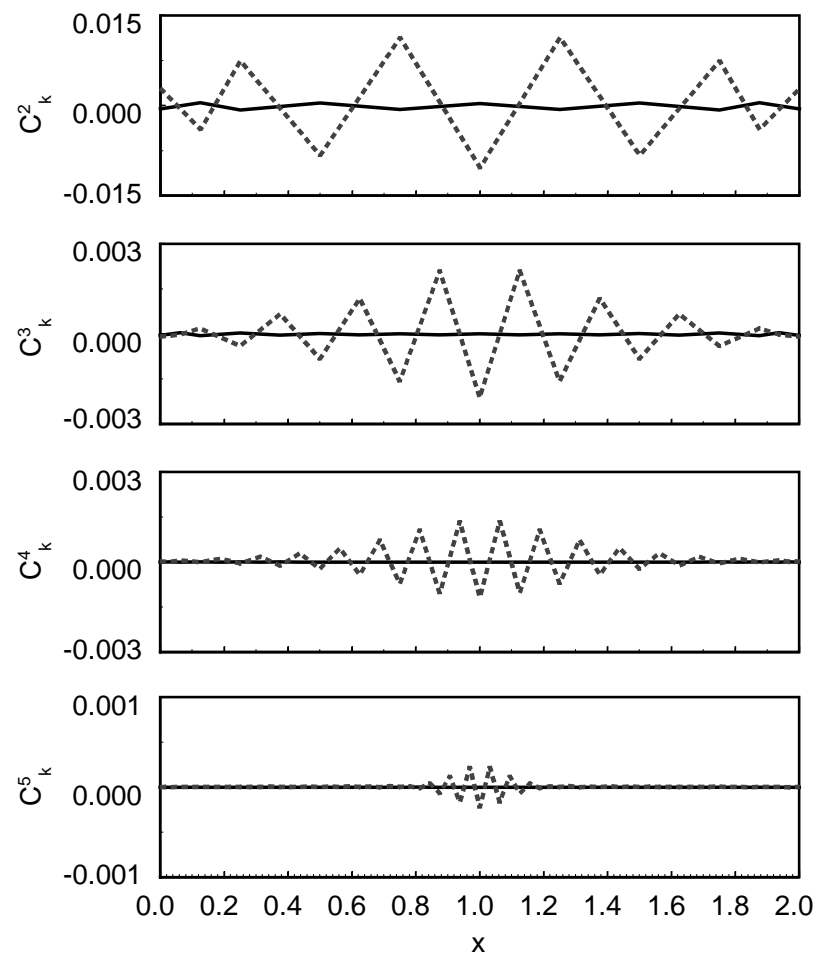

Fig. 2. Wavelet coefficients of a uniform string (solid line) and a string with a central concentrated mass $m=0.005$ (dashed line) at different levels of resolution.

Then we study the effect of the magnitude of the superimposed mass on the amplitude and width of the deviation region. We use the lower part of the envelope of the wavelet coefficients of the nonuniform string to represent the deviation region. Fig. 3 shows the 4 th level envelopes for various values of the attached mass. The amplitude of the deviation region is defined as the maximum value of the envelope, while the bandwidth of the deviation region is the distance between the half-power points of the envelope. Fig. 4 shows the amplitude of the deviation region at each level as a function of the superimposed mass. In the region of $0 \leqslant m \leqslant 0.03$, the amplitude is proportional to the superimposed mass. On the other hand, the bandwidth of the deviation region at each level is somewhat independent of the amount of the superimposed mass, as can be seen from Fig. 5. For a fixed value of the attached mass, both the amplitude and bandwidth decrease as the level of resolution increases.

Two mass points are attached to the uniform string for the investigation of the conditions under which two isolated mass points are discernible using the wavelet coefficients. The two concentrated masses, each of magnitude 0.05 , are located symmetrically with respect to the center of the string. Let $d$ denote the distance between the two mass points. Fig. 6 compares the 4th level wavelet coefficients of the deflections of the uniform string (solid line) and a string with two concentrated masses (dashed line) for various values of $d$. Figs. 6(a) and (b) show, respectively, the results for $d=1.0$ and 0.75 , both are much larger than the bandwidth of the 4 th level envelope. In these 


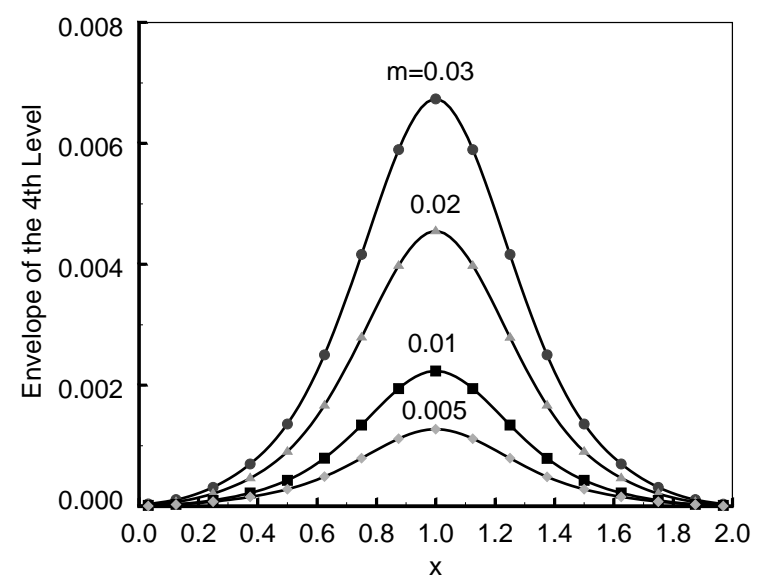

Fig. 3. Envelope of the 4th level deviation region for a uniform string with a central concentrated mass $m$ of various magnitudes.

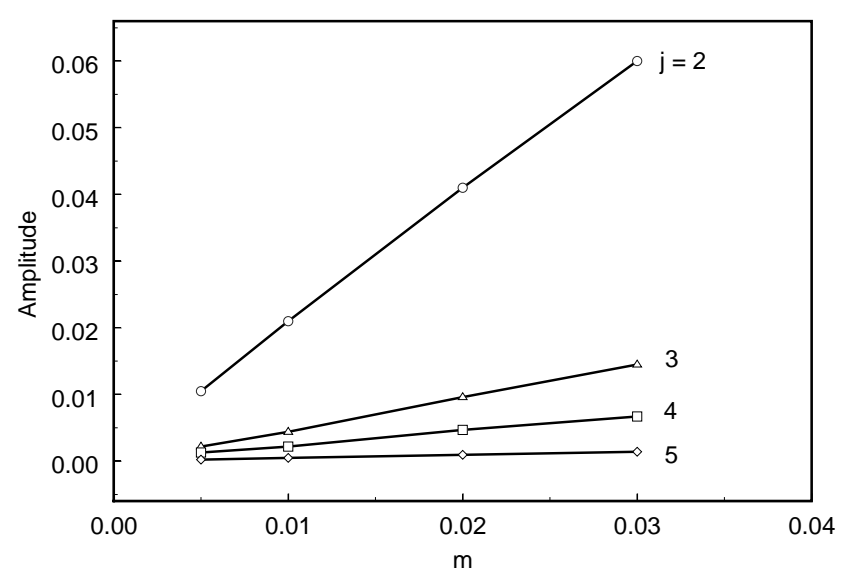

Fig. 4. Amplitude of the deviation region as a function of $m$ at different levels of resolution.

two cases, the deviation region seems to consist of two packets, each associated with one of the superimposed masses. Furthermore, the maximum amplitude of each packet occurs exactly at the location of the corresponding mass. If we reduce $d$ to 0.5 , which is close to the bandwidth of the 4th level envelope, the two packets converges in such a way that the deviation region looks like a single packet with a nearly flat top, as can be seen in Fig. 6(c). In this case we can still identify two mass points, but not as clearly as in cases (a) and (b). Then we further reduce $d$ to 0.25 , which is smaller than the bandwidth of the 4th level envelope. The results are shown in Fig. 6(d). Also shown for comparison are the wavelet coefficients of a uniform string with a single mass of magnitude 0.01 attached at the center, as indicated by the solid square dots. It is seen that the 4 th level wavelet coefficients induced by the two isolate mass points are indistinguishable from those due to a single mass point. In order to identify the two masses more clearly, we need to use a higher 


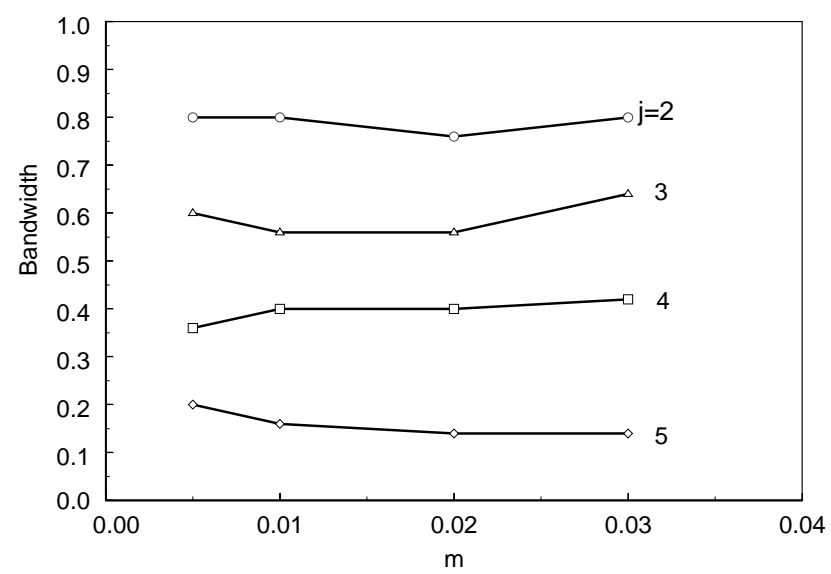

Fig. 5. Bandwidth of the deviation region as a function of $m$ at different levels of resolution.
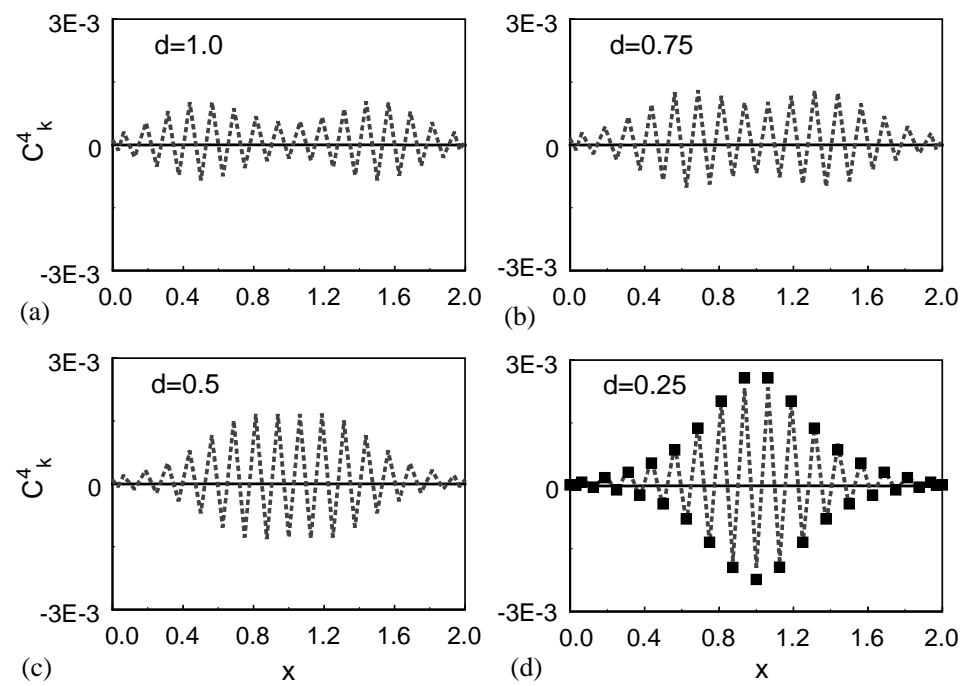

Fig. 6. Comparison of the 4th level wavelet coefficients of a uniform string (solid line) and a string with two mass points, each of magnitude 0.005 , attached symmetrically with respect to the center (dashed line). The solid dots in (d) indicate the wavelet coefficients of a string with a single mass of magnitude of 0.01 attached at the center.

level of resolution. Fig. 7 shows the 5th level wavelet coefficients. The bandwidth of the 5th level envelope due to a single mass is about 0.15 , which is smaller than the values of $d$ in all cases. In each case shown in Fig. 7, the deviation region is composed of two separate packets. Consequently, the two mass points can be identified clearly. These results indicate that two mass points can be distinguished if their separation is larger than the bandwidth of the deviation region. It follows that the resolving power increases with the level of resolution. However, the level of resolution cannot be increased without limit in practice. It is limited by the number of sensors available for measuring the vibration signals and the environmental noise. 

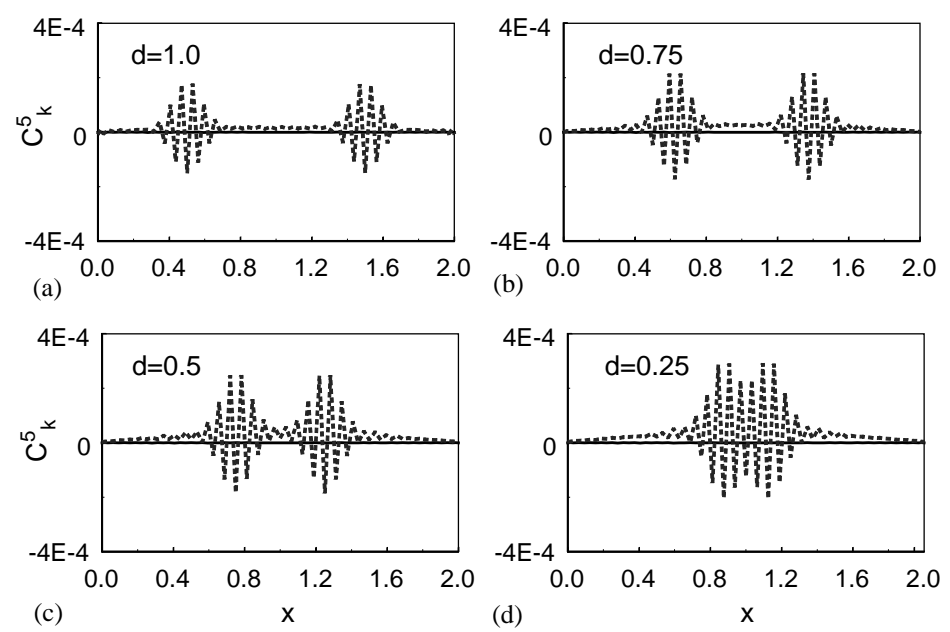

Fig. 7. Comparison of the 5th level wavelet coefficients of a uniform string (solid line) and a string with two mass points, each of magnitude 0.005 , attached symmetrically with respect to the center (dashed line).

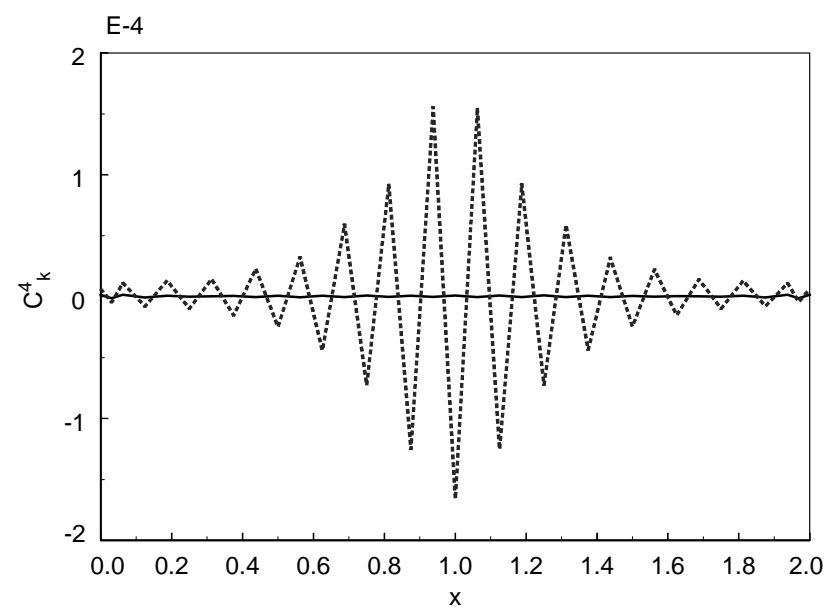

Fig. 8. Comparison of the 4th level wavelet coefficients of the first modes of a uniform string (solid line) and a string with a spring attached at the center (dashed line).

Mode shapes are often monitored for structural damage detection. For instance, Qian et al. [9] and Kam and Lee [10] measured the mode shapes of a cracked beam for the determination of the size of the crack. However, it may be difficult to detect the damage by simply comparing the mode shapes of the damaged and the original systems. A crack on an elastic structure introduces local flexibility due to the strain energy concentration in the vicinity of the crack tip under load. The local flexibility induced by the presence of the crack can be modeled appropriately by a spring. In this paper, we connect a spring to the center of the uniform string to simulate the effect of a crack. The stiffness of the spring is 0.01 . Wavelet transform is used to analyze the fundamental modes of the uniform string with and without a spring attached at the center. The results are shown in Fig. 8. We can see 
a clear "deviation region" in the figure and the maximum difference occurs at the location of the attached spring. Hence, small defects can be located precisely by monitoring the wavelet coefficients of the fundamental mode.

Our results indicate that a change of local density or flexibility due to damage will manifest itself as changes of the wavelet coefficients. Therefore, if the vibration signals can be measured accurately at the collocation points as required by the proposed method, changes of the wavelet coefficients of the measured vibration signals can be used to locate the defects on structures.

\section{Conclusions}

In this paper, we proposed a method based on the discrete wavelet transform for structural damage detection and identification. We used a uniform string to represent the original system; while a spring and some concentrated masses are employed to represent localized damaged parts. It is found that minor localized damage, which generates vibration signals almost indistinguishable from those of the undamaged system, can induce significant changes of the wavelet coefficients. The area where the difference between the wavelet coefficients is significant is called the deviation region. For a single mass point attached to the uniform string, the bandwidth of the deviation region is independent of the amount of the attached mass. Both the amplitude and bandwidth of the deviation region decrease as the level of resolution increases. Furthermore, the maximum value of the deviation region occurs in proximity to the attached mass. Two mass points can be distinguished clearly if their separation is larger than the bandwidth of the deviation region. A relatively weak spring is connected to the uniform string to model the minor local flexibility induced by a crack. Although the fundamental mode of the uniform string is almost indistinguishable from that of the string with an attached spring, differences between the wavelet coefficients of the fundamental modes are significant enough to indicate the existence of the attached spring. These results indicate that the location of the maximum value and the bandwidth of the deviation region can be used to identify and locate the damaged part.

\section{Acknowledgements}

This research is supported by the National Science Council of the Republic of China under Grant No. NSC88-2212-E-002-016.

\section{References}

[1] McFadden PD. Interpolation techniques for time domain averaging of gear vibration. Mechanical Systems and Signal Processing 1986;3:87-97.

[2] Lan MS, Naerheim Y. In-process detection of tool breakage in milling. ASME Journal of Engineering for Industry 1986;108:191-7.

[3] Wang WJ, McFadden PD. Early detection of gear failure by vibration analysis-I. Calculation of the time-frequency distribution. Mechanical Systems and Signal Processing 1993;7:193-203.

[4] Tsai TC, Wang YZ. Vibration analysis and diagnosis of a cracked shaft. Journal of Sound and Vibration 1996;192:607-20. 
[5] Ehrich FF. Sum and difference frequencies in vibration of high speed rotating machinery. ASME Journal of Engineering for Industry 1972;94:181-4.

[6] Cawley P, Adams RD. The location of defects in structures from measurements of natural frequencies. Journal of Strain Analysis 1979;14:49-57.

[7] Wauer J. On the dynamics of cracked rotors: a literature survey. Applied Mechanics Reviews 1990;43:13-7.

[8] Rizos PF, Aspragathos N, Dimarogonas AD. Identification of crack location and magnitude in a cantilever beam from the vibration modes. Journal of Sound and Vibration 1990;138:381-8.

[9] Qian GL, Gu SN, Jiang JS. The dynamic behavior and crack detection of a beam with a crack. Journal of Sound and Vibration 1990;138:233-43.

[10] Kam TY, Lee TY. Detection of cracks in structures using modal test data. Engineering Fracture Mechanics 1992;42:381-7.

[11] Baruh H, Ratan S. Damage detection in flexible structures. Journal of Sound and Vibration 1993;166:21-30.

[12] Dimarogonas AD, Papadopoulos CA. Vibration of cracked shaft in bending. Journal of Sound and Vibration 1983;91:583-93.

[13] Tansel IN, Mekdeci C, McLaughlin C. Detection of tool failure in end milling with wavelet transformations and neural networks. International Journal of Machine Tools and Manufacture 1995;35:1137-47.

[14] Mori K, Kasashima N, Yoshioka T, Ueno Y. Prediction of spalling on a ball bearing by applying the discrete wavelet transform to vibration signals. Wear 1996;195:162-8.

[15] Wang WJ, McFadden PD. Application of wavelets to gearbox vibration signals for fault detection. Journal of Sound and Vibration 1996;192:927-39.

[16] Boulahbal D, Colnaraghi MF, Ismail F. Amplitude and phase wavelet maps for the detection of cracks in geared systems. Mechanical Systems and Signal Processing 1999;13:423-36.

[17] Daubechies I. Ten lectures on wavelets. Philadelphia, PA, USA: SIAM, 1992.

[18] Vasilyev OV, Paolucci S, Sen M. A multilevel wavelet collocation method for solving partial differential equations in a finite domain. Journal of Computational Physics 1995;120:33-47.

[19] Vasilyev OV, Paolucci S. A dynamically adaptive multilevel wavelet collocation method for solving partial differential equations in a finite domain. Journal of Computational Physics 1996;125:498-512. 